\title{
The homotopy Gerstenhaber algebra of Hochschild cochains of a regular algebra is formal
}

\author{
Vasiliy Dolgushev, Dmitry Tamarkin, and Boris Tsygan
}

\begin{abstract}
The solution of Deligne's conjecture on Hochschild cochains and the formality of the operad of little disks provide us with a natural homotopy Gerstenhaber algebra structure on the Hochschild cochains of an associative algebra. In this paper we construct a natural chain of quasi-isomorphisms of homotopy Gerstenhaber algebras between the Hochschild cochain complex $C^{\bullet}(A)$ of a regular commutative algebra $A$ over a field $\mathbb{K}$ of characteristic zero and the Gerstenhaber algebra of multiderivations of $A$. Unlike the original approach of the second author based on the computation of obstructions our method allows us to avoid the bulky Gelfand-Fuchs trick and prove the formality of the homotopy Gerstenhaber algebra structure on the sheaf of polydifferential operators on a smooth algebraic variety, a complex manifold, and a smooth real manifold.
\end{abstract}

Mathematics Subject Classification (2000). 53D55.

Keywords. Hochschild cohomology, deformations, Gerstenhaber algebras.

\section{Introduction}

Various proofs of Deligne's Hochschild cohomological conjecture [21], [23], [26] [30] by J. E. McClure, J. H. Smith, M. Kontsevich, Y. Soibelman, A. Voronov and the second author show that the complex $C^{\bullet}(A)$ of Hochschild cochains of an associative algebra $A$ is equipped with a remarkable structure of a homotopy Gerstenhaber algebra. The homotopy Lie part of this structure coincides with the DGLA structure provided by the Gerstenhaber bracket [10] and the corresponding commutative (up to homotopy) product is cohomologous to the cup product.

If $A$ is the algebra of polynomials $\mathbb{K}\left[x_{1}, \ldots, x_{n}\right]$ over the field $\mathbb{K}$ of characteristic zero then it is not hard to prove formality of the homotopy Gerstenhaber algebra $C^{\bullet}(A)$ by explicitly computing the obstructions [16], [26].

In paper [14] G. Halbout showed that the structure maps of a $G_{\infty}$ quasi-isomorphism for the Hochschild cochains of the algebra $\mathbb{R}\left[\left[x_{1}, \ldots, x_{n}\right]\right]$ can be made relative to the Lie subalgebra $\mathrm{gl}_{n} \subset \operatorname{Der}\left(\mathbb{R}\left[\left[x_{1}, \ldots, x_{n}\right]\right]\right)$. This result suggests that 
the Gelfand-Fuchs trick [4], [6], [8], [9], [28], [32] could be applied to globalization of the $G_{\infty}$ quasi-isomorphism.

In this article we construct a natural chain of quasi-isomorphisms of homotopy Gerstenhaber algebras between the Hochschild cochain complex of a regular commutative algebra $A$ and the Gerstenhaber algebra of multiderivations of $A$. An important advantage of this approach in comparison to the original proof [16] and [26] of the second author is that we propose an explicit construction of a chain of quasi-isomorphisms, while in [16] and [26] only the existence of the desired chain is proved. This approach allows us to avoid the bulky Gelfand-Fuchs trick and prove the formality of the homotopy Gerstenhaber algebra structure on the sheaf of polydifferential operators on a smooth algebraic variety, a complex manifold, and a smooth real manifold.

The organization of this paper is as follows. In the introductory section we explain notation and recall basic facts about (co)algebras and (co)operads. In the second section we discuss properties of the homotopy Gerstenhaber algebra structure on the Hochschild cochain complex induced by the action [23] of the singular chains of the operad of little squares. In Section 3 we prove the main result of this paper, Theorem 4 , and in Section 4 we give obvious generalizations of this theorem. In the concluding section we discuss open questions and further possible applications of our results.

Acknowledgment. We are thankful to G. Halbout and M. Markl for discussions. D. T. and B. T. are supported by NSF grants. D. T. is also supported by the research fellowship of A.P. Sloan. The work of V. D. is partially supported the grant CRDF RM1-2545-MO-03 and the Grant for Support of Scientific Schools NSh-8065.2006.2.

1.1. Notation. Our underlying symmetric monoidal categories are the category of graded vector spaces and the category of chain complexes. Objects of these categories we sometimes loosely call "spaces". By suspension $\Sigma V$ of a space $V$ we mean $\varepsilon \otimes V$, where $\varepsilon$ is a one-dimensional vector space placed in degree +1 . The underlying field $\mathbb{K}$ has characteristic zero.

For an operad $\mathcal{O}$ we denote by $\operatorname{Alg}_{\mathcal{O}}$ the category of algebras over the operad $\mathcal{O}$. Dually, for a cooperad $\mathcal{C}$ we denote by $\mathrm{Coalg}_{\mathcal{C}}$ the category of nilpotent ${ }^{1}$ coalgebras over the cooperad $\mathcal{C}$. By corestriction we mean the canonical map

$$
u_{V}: \mathbb{F}_{e}(V) \rightarrow V
$$

from the cofree coalgebra $\mathbb{F}_{\mathcal{C}}(V)$ to the space of its cogenerators $V$. $\operatorname{Ho}(\mathcal{O})$ is reserved for the operad of homotopy $\mathcal{O}$-algebras.

For a quadratic operad $\mathcal{O}$ we will denote by $\mathcal{O}^{\vee}$ the Koszul dual cooperad [7], [13]. $\operatorname{Bar}(\mathcal{O})$ will denote the bar construction of the (augmented) operad $\mathcal{O}$, and $\operatorname{Cobar}(\mathcal{C})$ will denote the cobar construction of the (coaugmented) cooperad $\mathcal{C}$. For an operad $\mathcal{O}\left(\right.$ resp. cooperad $\mathcal{C}$ ) and a space $V$ we denote by $\mathbb{F}_{\mathcal{O}}(V)\left(\right.$ resp. by $\left.\mathbb{F}_{\mathcal{C}}(V)\right)$ the free

\footnotetext{
${ }^{1}$ For the definition of nilpotent coalgebra see Section 2.4.1 in [16].
} 
algebra (resp. cofree coalgebra) over the operad $\mathcal{O}(\operatorname{resp}$. cooperad $\mathcal{C})$. We will use the following list of (co)operads:

- assoc (resp. coassoc) is the operad of associative algebras without unit (resp. the cooperad of coassociative algebras without counit).

- lie (resp. colie) is the operad of Lie algebras (resp. the cooperad of Lie coalgebras).

- comm (resp. cocomm) is the operad of commutative (associative) algebras (resp. the operad of cocommutative coassociative coalgebras).

- $\mathbf{e}_{2}$ denotes the operad of Gerstenhaber algebras.

- Braces denotes the operad of braces [11], [18]. The definition of this operad is given $^{2}$ in paper [23] by J. E. McClure and J. H. Smith. In their paper it is denoted by $\mathscr{H}$.

- lie $^{+}$(resp. colie $\left.{ }^{+}\right)$denotes the 2-colored operad of pairs "Lie algebra + its module" (resp. the 2-colored cooperad of pairs "Lie coalgebra + its comodule").

- comm $^{+}$(resp. cocomm ${ }^{+}$) denotes the 2-colored operad of pairs "commutative algebra + its module" (resp. the 2-colored cooperad of pairs "cocommutative coalgebra + its comodule").

By "suspension" of a (co)operad $\mathcal{O}$ we mean the (co)operad $\Lambda(\mathcal{O})$ whose $m$-th space is

$$
\Lambda(\mathcal{O})(m)=\Sigma^{1-m} \mathcal{O}(m) \otimes \operatorname{sgn}_{m},
$$

where $\operatorname{sgn}_{m}$ is the sign representation of the symmetric group $S_{m}$.

For a commutative algebra $\mathcal{B}$ and a $\mathcal{B}$-module $\mathcal{V}$ we denote by $S_{\mathscr{B}}(\mathcal{V})$ the symmetric algebra of $\mathcal{V}$ over $\mathscr{B} . S_{\mathscr{B}}^{m}(\mathcal{V})$ stands for the $m$-th component of this algebra. If $\mathcal{B}=\mathbb{K}$ then $\mathcal{B}$ is omitted from the notation.

For an associative algebra $A$

$$
C^{\bullet}(A)=\operatorname{Hom}\left(A^{\otimes \bullet}, A\right)
$$

denotes the normalized Hochschild cochain complex. $[,]_{G}$ and $\cdot$ denote the Gerstenhaber bracket and the cup product in $C^{\bullet}(A)$, respectively.

1.2. Preliminaries. In this section we recall some basic facts about (co)algebras and (co)operads we will use in the paper.

\footnotetext{
${ }^{2}$ See the paragraph on page 3 right above Theorem 1.1 in [23].
} 
1.2.1. Koszul (co)operads and their (co)algebras. Let us recall [13] that a quadratic operad $\mathcal{O}$ is Koszul if the canonical map

$$
\operatorname{Cobar}\left(\mathcal{O}^{\vee}\right) \rightarrow \mathcal{O}
$$

is a quasi-isomorphism.

For any coaugmented cooperad $\mathcal{C}$ the operad Cobar $(\mathcal{C})$ is obviously cofibrant [15]. Thus, as well as Koszul algebras [25], Koszul operads [13] admit simpler cofibrant resolutions.

This observation motivates the following definition:

Definition 1. For a Koszul operad $\mathcal{O}$ the operad $\operatorname{Cobar}\left(\mathcal{O}^{\vee}\right)$ can be viewed as an operad $\operatorname{Ho}(\mathcal{O})$ of homotopy $\mathcal{O}$-algebras.

Let us also recall that for any coaugmented cooperad $\ell$ the algebras over the operad Cobar( $\mathcal{C}$ ) have the following explicit description due to [12]:

Proposition 1 (Proposition 2.15, [12]). Let $C$ be a coaugmented cooperad and let $V$ be a space. There is a natural bijection between the degree 1 codifferentials on the cofree coalgebra $\mathbb{F}_{\mathcal{C}}(V)$ and the Cobar(C)-algebra structures on $V$.

Furthermore, one can describe coderivations of a cofree coalgebra.

Proposition 2 (Proposition 2.14 [12]). Let $C$ be a cooperad and let $\operatorname{Coder}\left(\mathbb{F}_{e}(V)\right)$ denote the Lie algebra of coderivations of the cofree coalgebra $\mathbb{F}_{e}(V)$. Then the composition with the corestriction $u_{V}: \mathbb{F}_{e}(V) \rightarrow V$ induces an isomorphism

$$
\operatorname{Coder}\left(\mathbb{F}_{e}(V)\right) \cong \operatorname{Hom}\left(\mathbb{F}_{e}(V), V\right)
$$

of the graded vector space of coderivations of the cofree coalgebra $\mathbb{F}_{e}(V)$ with the graded vector space $\operatorname{Hom}\left(\mathbb{F}_{e}(V), V\right)$.

In view of Definition 1, Proposition 1 implies the following.

Corollary 1. For a Koszul operad $\mathcal{O}$ and a space $V$ there is a natural bijection between the $\mathrm{Ho}(\mathcal{O})$-algebra structures on $V$ and degree 1 codifferentials on the cofree coalgebra $\mathbb{F}_{\mathcal{O} \vee}(V)$.

The above corollary motivates the definition of homotopy morphisms between $\operatorname{Ho}(\mathcal{O})$-algebras for a Koszul operad $\mathcal{O}$ :

Definition 2. A homotopy morphism $F$ from a $\mathrm{Ho}(\mathcal{O})$-algebra $V_{1}$ to a $\mathrm{Ho}(\mathcal{O})$-algebra $V_{2}$ is a morphism of the DG coalgebras

$$
F:\left(\mathbb{F}_{\mathcal{O} \vee}\left(V_{1}\right), d_{V_{1}}\right) \rightarrow\left(\mathbb{F}_{\mathcal{O} \vee}\left(V_{2}\right), d_{V_{2}}\right),
$$


where $d_{V_{1}}$ and $d_{V_{2}}$ are codifferentials corresponding to the $\mathrm{Ho}(\mathcal{O})$-algebra structures on $V_{1}$ and $V_{2}$, respectively.

We reserve the following notation for such morphisms

$$
F: V_{1} \succ V_{2} \text {. }
$$

Let $u$ and $\lambda$ be, respectively, the corestriction (1.1) and the coaugmentation of the cooperad $\mathcal{O}^{\vee}$. Similarly to Proposition 2 one can show that every morphism of cofree coalgebras

$$
F: \mathbb{F}_{\mathcal{O} \vee}\left(V_{1}\right) \rightarrow \mathbb{F}_{\mathcal{O} \vee}\left(V_{2}\right)
$$

is uniquely determined by its composition with the corestriction

$$
u_{V_{2}}: \mathbb{F}_{\mathcal{O} \vee}\left(V_{2}\right) \rightarrow V_{2}
$$

In what follows we call the components

$$
F_{n}=\left.u_{V_{2}} \circ F\right|_{\mathcal{O}^{\vee}(n) \otimes S_{n} V_{1}^{\otimes n}} \mathcal{O}^{\vee}(n) \otimes_{S_{n}} V_{1}^{\otimes n} \rightarrow V_{2}
$$

of the composition $u_{V_{2}} \circ F$ the structure maps of the homotopy morphism $F$ (1.5).

Using Definition 2 it is not hard to show that the first structure map (1.7) of (1.5)

$$
F_{1}=u_{V_{2}} \circ F \circ \lambda: V_{1} \rightarrow V_{2}
$$

is always a morphism of the corresponding complexes. This observation motivates the following definition:

Definition 3. Let $\mathcal{O}$ be a Koszul operad. A quasi-isomorphism between $\mathrm{Ho}(\mathcal{O})$ algebras $V_{1}$ and $V_{2}$ is a homotopy morphism (1.5) whose first structure map (1.8) is a quasi-isomorphism of complexes.

We will often have to deal with (co)free (co)algebras over a suspended (co)operad. The following easy fact will be very useful:

$$
\mathbb{F}_{\Lambda \mathcal{O}}(V)=\Sigma \mathbb{F}_{\mathcal{O}}\left(\Sigma^{-1} V\right)
$$

Equation (1.9) holds both for operads and cooperads.

Let $\mathcal{O}$ be an augmented operad and let $\mathcal{O}^{\vee}$ be its Koszul dual cooperad. Then according to [12] we have

Theorem 1 (Theorem 2.25, [12]). There is an adjunction

$$
\Omega_{\mathcal{O}}: \operatorname{Coalg}_{\mathcal{O} \vee} \rightleftharpoons \operatorname{Alg}_{\mathcal{O}}: B_{\mathcal{O} \vee}
$$


between the category $\operatorname{Coalg}_{\mathcal{O}} \vee$ of nilpotent $\mathcal{O}^{\vee}$-coalgebras and the category $\operatorname{Alg}_{\mathcal{O}}$ of $\mathcal{O}$-algebras. If $\mathcal{O}$ is Koszul the counit

$$
\eta_{\mathcal{O}}: \Omega_{\mathcal{O}}\left(B_{\mathcal{O}} \vee(A)\right) \rightarrow A
$$

and the unit

$$
\varepsilon_{\mathcal{O} \vee}: C \rightarrow B_{\mathcal{O} \vee}\left(\Omega_{\mathcal{O}}(C)\right)
$$

of this adjunction are quasi-isomorphisms.

Remark 1. Notice that if $A$ is an $\mathcal{O}$-algebra, then as a coalgebra $B_{\mathcal{O}} \vee(A)$ is just the cofree coalgebra $\mathbb{F}_{\mathcal{O}} \vee(A)$ cogenerated by $A$. Dually, if $C$ is a $\mathcal{O}^{\vee}$-coalgebra then as an algebra $\Omega_{\mathcal{O}}(C)$ is $\mathbb{F}_{\mathcal{O}}(C)$. Thus the algebra structure on $A$ (resp. the coalgebra structure on $C$ ) contributes only to the codifferential on $B_{\mathcal{O}^{\vee}}(A)$ (resp. differential on $\Omega_{\mathcal{O}}(C)$ ).

Remark 2. An obvious analogue of Theorem 1 can be proved for colored operads.

The counit (1.11) and the unit (1.12) of the adjunction (1.10) admit simple explicit descriptions. ${ }^{3}$ To describe the map $\eta_{\mathcal{O}}(1.11)$ we notice that it is a map of $\mathcal{O}$-algebras from a free $\mathcal{O}$-algebra. Hence, $\eta_{\mathcal{O}}$ is uniquely determined by its restriction to the space of generators. Using the definition of the functors $\Omega_{\mathcal{O}}$ and $B_{\mathcal{O}^{\vee}}$ [12] it is not hard to see that

$$
\left.\eta_{\mathcal{O}}\right|_{B_{\mathcal{O}} \vee(A)}=u_{A}
$$

where $u_{A}$ is the corestriction

$$
u_{A}: \mathbb{F}_{\mathcal{O} \vee}(A) \rightarrow A .
$$

Similarly, the map $\varepsilon_{\mathcal{O}} \vee$ being a map to a cofree $\mathcal{O}^{\vee}$-coalgebra is uniquely determined by its composition $u_{\mathbb{F}_{\mathcal{O}}(C)} \circ \varepsilon_{\mathcal{O}} \vee$ with the corestriction

$$
u_{\mathbb{F}_{\mathcal{O}}(C)}: \mathbb{F}_{\mathcal{O}} \vee\left(\mathbb{F}_{\mathcal{O}}(C)\right) \rightarrow \mathbb{F}_{\mathcal{O}}(C)
$$

onto cogenerators.

Again, using the definition of the functors $\Omega_{\mathcal{O}}$ and $B_{\mathcal{O}} \vee$ [12] one can show that

$$
u_{\mathbb{F}_{\mathcal{O}}(C)} \circ \varepsilon_{\mathcal{O}} \vee=\rho,
$$

where $\rho$ is the embedding of generators to the free algebra:

$$
\rho: C \rightarrow \mathbb{F}_{\mathcal{O}}(C) .
$$

\footnotetext{
${ }^{3}$ See Sections 2.3 and 2.4 in [12].
} 
Let us now prove that if $\mathcal{O}$ is a Koszul operad then for every $\operatorname{Ho}(\mathcal{O})$-algebra $V$ one can construct a DG $\mathcal{O}$-algebra which is quasi-isomorphic ${ }^{4}$ to the initial $\operatorname{Ho}(\mathcal{O})$ algebra $V$.

To construct this algebra we first notice that, due to corollary 1 , a $\operatorname{Ho}(\mathcal{O})$-algebra $V$ corresponds to a degree 1 codifferential $D$ of the cofree $\mathcal{O}^{\vee}$-coalgebra $\mathbb{F}_{\mathcal{O}} \vee(V)$. Thus a $\operatorname{Ho}(\mathcal{O})$-algebra $V$ gives us the DG $\mathcal{O}^{\vee}$-coalgebra

$$
\left(\mathbb{F}_{\mathcal{O}} \vee(V), D\right) .
$$

Applying Theorem 1 to the above coalgebra we get the following morphism of DG coalgebras

$$
\chi^{V}=\varepsilon_{\mathcal{O}^{\vee}}:\left(\mathbb{F}_{\mathcal{O}^{\vee}}(V), D\right) \rightarrow B_{\mathcal{O} \vee}\left(\Omega_{\mathcal{O}}\left(\mathbb{F}_{\mathcal{O}^{\vee}}(V), D\right)\right) .
$$

Definition 2 allows us to treat (1.15) as a homotopy morphism from $V$ to $\Omega_{\mathcal{O}}\left(\mathbb{F}_{\mathcal{O}} \vee(V), D\right)$. The following proposition shows that this homotopy morphism is a quasi-isomorphism in the sense of Definition 3.

Proposition 3. Let $\mathcal{O}$ be a Koszul operad and $D$ be a codifferential on the cofree $\mathcal{O}^{\vee}$-coalgebra $\mathbb{F}_{\mathcal{O}} \vee(V)$ defining a $\mathrm{Ho}(\mathcal{O})$-algebra structure on a space $V$. Then the homotopy morphism $\chi^{V}$,

$$
\chi^{V}: V \succ \Omega_{\mathcal{O}}\left(\mathbb{F}_{\mathcal{O}^{\vee}}(V), D\right),
$$

corresponding to the map (1.15) is a quasi-isomorphism.

Proof. Let, as above, $u$ and $\lambda$ denote, respectively, the corestriction (1.1) and the coaugmentation of the cooperad $\mathcal{O}^{\vee}$.

According to Definition 3 we have to prove that the composition

$$
\chi_{1}^{V}=u \circ \chi^{V} \circ \lambda: V \rightarrow \Omega_{\mathcal{O}}\left(\mathbb{F}_{\mathcal{O}^{\vee}}(V), D\right)
$$

is a quasi-isomorphism of the corresponding complexes, where the differential on $V$ is the composition

$$
D_{1}=u \circ D \circ \lambda \text {. }
$$

Due to equation (1.14) the composition $u \circ \chi_{V}$ is simply the embedding

$$
\mathbb{F}_{\mathcal{O}} \vee(V) \hookrightarrow \mathbb{F}_{\mathcal{O}}\left(\mathbb{F}_{\mathcal{O}} \vee(V)\right)
$$

of the space $\mathbb{F}_{\mathcal{O}} \vee(V)$ of generators to the free $\mathcal{O}$-algebra $\mathbb{F}_{\mathcal{O}}\left(\mathbb{F}_{\mathcal{O}} \vee(V)\right)$. Hence, $\chi_{1}^{V}$ coincides with the embedding of $V$ into $\Omega_{\mathcal{O}}\left(\mathbb{F}_{\mathcal{O}} \vee(V), D\right)$. In particular, the graded vector space $V$ with the differential $D_{1}(1.17)$ is a subcomplex of $\Omega_{\mathcal{O}}\left(\mathbb{F}_{\mathcal{O} \vee}(V), D\right)$.

\footnotetext{
${ }^{4} \mathrm{An}$ analogous construction in topology is called rectification [1].
} 
It remains to prove that the quotient complex of the complex $\Omega_{\mathcal{O}}\left(\mathbb{F}_{\mathcal{O}} \vee(V), D\right)$ by the subcomplex $\left(V, D_{1}\right)$ is acyclic. Using the filtration corresponding to the total degree of the space $\mathbb{F}_{\mathcal{O}} \vee(V)$ it is not hard to reduce the latter question to the computation of the homology groups of the cofree $\mathcal{O}^{\vee}$-coalgebra $\mathbb{F}_{\mathcal{O}} \vee(V)$. Then the desired statement follows easily from the criterion of Ginzburg and Kapranov [13] (Theorem 4.2.5) and the koszulity of the operad $\mathcal{O}$.

1.2.2. Operads lie, comm, $\mathbf{e}_{2}$, et cetera. Let us recall the following well-known facts.

Proposition 4 (V. Ginzburg and M. Kapranov, [13]). The operads lie and comm are Koszul. We have

$$
\operatorname{lie}^{\vee}=\Lambda(\text { cocomm }), \quad \operatorname{comm}^{\vee}=\Lambda(\text { colie })
$$

Proposition 5 (E. Getzler and J. D. S. Jones, [12]). The operad $\mathbf{e}_{2}$ is Koszul.

Remark. In [12] E. Getzler and J. D. S. Jones proved koszulity for wider class of operads which includes the operad $\mathbf{e}_{2}$ of Gerstenhaber algebras. In fact, using the Ginzburg-Kapranov criterion of koszulity (see [13], Theorem 4.2.5) it is not hard to prove Proposition 5. This proof can be found in [16] (see Subsection 5.4.6).

A simple computation shows that coalgebras over $\mathbf{e}_{2}^{\vee}$ are equipped with a cocommutative coassociative coproduct of degree -2 and a Lie cobracket of degree -1 , satisfying the Leibniz rule. Thus

$$
\mathbb{F}_{\mathbf{e}_{2} \vee}(V)=\Sigma^{2} \mathbb{F}_{\text {cocomm }}\left(\Sigma^{-1} \mathbb{F}_{\text {colie }}\left(\Sigma^{-1} V\right)\right),
$$

and due to Corollary 1 and Proposition 5 the homotopy Gerstenhaber algebra structures on a space $V$ are in a one-to-one correspondence with the degree 1 codifferentials of the coalgebra (1.19).

Let $\left(V,[,]_{V}, \bullet\right)$ be a Gerstenhaber algebra with the bracket $[,]_{V}$ and the product $\bullet V$. Using the fact that $V$ is both a commutative algebra and a $\Lambda$ lie-algebra one can split the construction of the DG $\mathbf{e}_{2}{ }^{\vee}$-coalgebra $B_{\mathbf{e}_{2} \vee}(V)$ into two steps.

In the first step we upgrade the DG $\Lambda$ colie-coalgebra $B_{\Lambda \text { colie }}\left(V,{ }^{\cdot} V\right)$ to a DG Lie bialgebra (with the existing cobracket of degree 1 a Lie bracket [, ] of degree -1) using the bracket $[,]_{V}$ on $V$. The bracket $[$,$] on B_{\Lambda \text { colie }}\left(V, \bullet_{V}\right)$ is uniquely determined by the formula

$$
u_{V}([X, Y])=\left[u_{V}(X), u_{V}(Y)\right]_{V}, \quad X, Y \in B_{\Lambda \text { colie }}\left(V, \bullet_{V}\right)
$$

and the compatibility condition with the existing cobracket. Here, as above, $u_{V}$ denotes the corestriction $u_{V}: B_{\Lambda \text { colie }}(V) \rightarrow V$ on the space $V$ of cogenerators. The 
resulting bracket $[$,$] is compatible with the codifferential on B_{\Lambda \text { colie }}\left(V, \bullet_{V}\right)$ due to the Leibniz rule for the bracket $[,]_{V}$ on $V$.

In the second step we now apply the functor $B_{\Lambda^{2}}$ cocomm to the Lie bialgebra $B_{\Lambda \text { colie }}\left(V, \bullet_{V}\right)$, regarding this Lie bialgebra as a DG $\Lambda$ lie-algebra, and so obtain a DG $\Lambda^{2}$ cocomm-coalgebra

$$
C=B_{\Lambda^{2} \text { cocomm }} B_{\Lambda \text { colie }}(V, \bullet V) .
$$

Then extending in the similar way the Lie cobracket from $B_{\Lambda \text { colie }}\left(V,{ }^{\circ} V\right)$ to $C$ using the compatibility with the coproduct on $C$ we get the desired DG $\mathbf{e}_{\mathbf{2}}{ }^{\vee}$-coalgebra

$$
B_{\mathbf{e}_{2} \vee}(V)=B_{\Lambda^{2} \text { cocomm }} B_{\Lambda \text { colie }}(V, \cdot V) .
$$

The compatibility of the resulting cobracket on $C$ (1.21) with the codifferential follows from the compatibility of the cobracket and the bracket on $B_{\Lambda \text { colie }}(V, \bullet V)$.

The above construction of the DG $\mathbf{e}_{2}{ }^{\vee}$-coalgebra (1.22) shows that the functors $B_{\Lambda \text { colie }}$ and $B_{\Lambda^{2} \text { cocomm }}$ can be extended to functors

$$
B_{\Lambda \text { colie }}^{\prime}: \operatorname{Alg}_{\mathbf{e}_{2}} \rightarrow \text { Bialg }
$$

and

$$
B_{\Lambda^{2} \text { cocomm }}^{\prime}: \text { Bialg } \rightarrow \text { Coalg }_{\mathbf{e}_{2} \vee}
$$

where Bialg is the category of DG Lie bialgebras with the bracket of degree -1 and the nilpotent cobracket of degree 1 . The composition of these two functors gives us the functor $B_{\mathbf{e}_{2}} \vee$

$$
B_{\mathbf{e}_{2} \vee}=B_{\Lambda^{2} \text { cocomm }}^{\prime} \circ B_{\Lambda \text { colie }}^{\prime} .
$$

In what follows we will omit the prime in the notations of the functors (1.23) and (1.24).

It is also clear from (1.19) that there are canonical maps of operads:

$$
\operatorname{Ho}(\Lambda(\text { lie })) \rightarrow \operatorname{Ho}\left(\mathbf{e}_{2}\right), \quad \operatorname{Ho}(\mathbf{c o m m}) \rightarrow \operatorname{Ho}\left(\mathbf{e}_{2}\right) .
$$

We use the following realization of the cofree Lie coalgebra

$$
\mathbb{F}_{\text {colie }}(V)=\mathbb{F}_{\text {coassoc }}(V) / \mathbb{F}_{\text {coassoc }}(V) \cdot \bullet_{\text {sh }} \mathbb{F}_{\text {coassoc }}(V),
$$

where $\cdot$ sh denotes the shuffle product

$$
\begin{aligned}
\left\langle a_{1}, a_{2}, \ldots, a_{p}\right\rangle \cdot{ }_{\mathrm{sh}}\left\langle a_{p+1}, a_{p+2}, \ldots, a_{p+q}\right\rangle & \\
= & \sum_{\varepsilon \in \operatorname{Sh}(p, q)}\left\langle a_{\varepsilon^{-1}(1)}, a_{\varepsilon^{-1}(2)}, \ldots, a_{\varepsilon^{-1}(p+q)}\right\rangle, \quad a_{i} \in V .
\end{aligned}
$$

Here $\operatorname{Sh}(p, q)$ is the set of $(p, q)$-shuffles.

Let us conclude this section with the following statement.

Proposition 6. The operad comm ${ }^{+}$of pairs "commutative algebra + its module" is Koszul. 
Proof. It is not hard to see that

$$
\left(\operatorname{comm}^{+}\right)^{\vee}=\Lambda \text { colie }^{+} .
$$

Hence, due to a proper version of Ginzburg-Kapranov criterion (Theorem 4.2.5 in [13]) it suffices to show that Harrison homology groups of the free commutative algebra with trivial coefficients and with coefficients in itself are concentrated in the lowest degrees. Due to the realization (1.27) the latter question reduces to a simple computation with Hochschild chain complexes of the free commutative algebra.

\section{The Ho(e $\left.e_{2}\right)$-algebra structure on the Hochschild complex}

It follows from the result of [23] and the formality of the operad of little squares [27] that we have a quasi-isomorphism

$$
\mathrm{Ho}\left(\mathbf{e}_{2}\right) \stackrel{\sim}{\longrightarrow} \text { Braces }
$$

from the operad $\operatorname{Ho}\left(\mathbf{e}_{2}\right)$ of homotopy Gerstenhaber algebras to the operad of braces Braces.

This result implies that the normalized Hochschild complex $C^{\bullet}(A)$ of any associative algebra $A$ is naturally a $\mathrm{Ho}\left(\mathbf{e}_{2}\right)$-algebra. In what follows we say the $\mathrm{Ho}\left(\mathbf{e}_{2}\right)$ algebra structure on $C^{\bullet}(A)$ referring to the structure provided by the map (2.1). In this section we discuss properties of this $\mathrm{Ho}\left(\mathbf{e}_{2}\right)$-algebra.

The first remarkable property of the homotopy Gerstenhaber algebra $C^{\bullet}(A)$ can be formulated as

Theorem 2 ([26]). The $\operatorname{Ho}\left(\Lambda\left(\right.\right.$ lie))-algebra structure on $C^{\bullet}(A)$ induced by the first map in (1.26) coincides with the $D G \Lambda$ (lie)-algebra structure given by the Hochschild differential and the Gerstenhaber bracket.

Proof. If $\mathcal{O}$ is a Koszul operad then so is $\Lambda \mathcal{O}$ and $\Lambda^{-1} \mathcal{O}$. Furthermore, it is easy to see that the suspension commutes with the operation of taking the Koszul dual (co)operad:

$$
(\Lambda(\mathcal{O}))^{\vee}=\left(\Lambda(\mathcal{O})^{\vee}\right) .
$$

Thus the $\operatorname{Ho}(\Lambda($ lie $))$-algebra structure on $C^{\bullet}(A)$ is determined by a degree 1 map

$$
Q: \mathbb{F}_{\Lambda^{2} \text { cocomm }}\left(C^{\bullet}(A)\right) \rightarrow C^{\bullet}(A)
$$

or, in other words, by an infinite collection of maps

$$
Q_{m}: S^{m}\left(C^{\bullet}(A)\right) \rightarrow C^{\bullet}(A)
$$

of degree $3-2 m$. 
To prove the theorem we need to show that

$$
Q_{m}=0, \quad m>2
$$

and

$$
Q_{2}\left(P_{1}, P_{2}\right)=(-)^{\left|P_{1}\right|}\left[P_{1}, P_{2}\right]_{G} \text { for all } P_{i} \in C^{\bullet}(A),
$$

where $[,]_{G}$ is the Gerstenhaber bracket [10].

To prove (2.2) we notice that the lowest degree $m$-ary operation we can get by combining the braces and the cup product is

$$
\left(P_{1}, \ldots, P_{m}\right) \rightarrow P_{i}\left\{P_{1}, \ldots, \widehat{P}_{i}, \ldots, P_{m}\right\},
$$

where $i$ runs from 1 to $m$ and $P_{i} \in C^{\bullet}(A)$.

It is easy to see that the degree of the operation (2.4) is $1-m$. Thus if $m>2$ then the degree of $Q_{m}$ is lower than the degree that can be obtained by combining the braces and the cup-product. Hence for any $m>2, Q_{m}=0$.

Since on the cohomology space $H^{\circ}(A)$ both $\operatorname{Ho}\left(\mathbf{e}_{2}\right)$-algebras induce the same graded Lie algebra structure we conclude that for any pair $P_{1}, P_{2} \in C^{\bullet}(A)$

$$
Q_{2}\left(P_{1}, P_{2}\right)=(-)^{\left|P_{1}\right|}\left[P_{1}, P_{2}\right]_{G}+\partial \Psi\left(P_{1}, P_{2}\right)-\Psi\left(\partial P_{1}, P_{2}\right)-(-)^{\left|P_{1}\right|} \Psi\left(P_{1}, \partial P_{2}\right),
$$

where $\partial$ is the Hochschild coboundary operator and

$$
\Psi: C^{\bullet}(A) \otimes C^{\bullet}(A) \rightarrow C^{\bullet}(A)
$$

is a map of degree -2 expressed in terms of braces and the cup-product.

Using the braces and the cup-product it is impossible to get a binary operation of degree -2 . This observation implies equation (2.3) and concludes the proof.

Due to Corollary 1 and Proposition 5 the $\operatorname{Ho}\left(\mathbf{e}_{2}\right)$-algebra on $C^{\bullet}(A)$ is encoded by a degree 1 codifferential

$$
M: \mathbb{F}_{\mathbf{e}_{2} \vee}\left(C^{\bullet}(A)\right) \rightarrow \mathbb{F}_{\mathbf{e}_{2} \vee}\left(C^{\bullet}(A)\right)
$$

of the cofree $\mathbf{e}_{2}{ }^{\vee}$-coalgebra $\mathbb{F}_{\mathbf{2}_{2} \vee}\left(C^{\bullet}(A)\right)$ cogenerated by $C^{\bullet}(A)$.

Due to Proposition 2, $M$ is uniquely determined by the composition $u_{C}{ }^{\bullet}(A) \circ M$ with the corestriction

$$
u_{C^{\bullet}(A)}: \mathbb{F}_{\mathbf{e}_{2} \vee}\left(C^{\bullet}(A)\right) \rightarrow C^{\bullet}(A) .
$$

We denote this composition $u_{C^{\bullet}(A)} \circ M$ by $m$,

$$
m=u_{C^{\bullet}(A)} \circ M: \Sigma^{2} \mathbb{F}_{\text {cocomm }}\left(\Sigma^{-1} \mathbb{F}_{\text {colie }}\left(\Sigma^{-1} C^{\bullet}(A)\right)\right) \rightarrow C^{\bullet}(A) .
$$

Like (2.6) the map (2.8) is of degree 1. 
From now on we will assume that $A$ is a regular commutative algebra. Let $\operatorname{Der}(A)$ denote the Lie algebra of derivations of $A$ and let

$$
V^{\bullet}(A)=S_{A}^{\bullet}(\Sigma \operatorname{Der}(A)) .
$$

be the Gerstenhaber algebra of polyvectors on $\operatorname{Spec}(A)$.

It is well known [17] that the natural embedding

$$
V^{\bullet}(A) \rightarrow C^{\bullet}(A)
$$

is a quasi-isomorphism of complexes where $V^{\bullet}(A)$ is viewed as a complex with the vanishing differential. In other words, $V^{\bullet}(A)$ is the cohomology space of the Hochschild cochain complex $\left(C^{\bullet}(A), \partial\right)$. In [17] (see Theorem 5.2) it was shown that the product induced on the cohomology space $V^{\bullet}(A)$ coincides with natural product on (2.9). Furthermore, it is not hard to show that the $\Lambda$ lie-algebra structure induced on the cohomology space $V^{\bullet}(A)$ coincides with the one given by the Schouten-Nijenhuis bracket [24].

Let us introduce the following sub $\mathbf{e}_{2}{ }^{\vee}$-coalgebra

$$
\begin{gathered}
\Xi(A) \subset \mathbb{F}_{\mathbf{e}_{2} \vee}\left(C^{\bullet}(A)\right), \\
\Xi(A)=\mathbb{F}_{\Lambda^{2} \text { cocomm }} \circ \mathbb{F}_{\Lambda \text { colie }^{+}}(A, \Sigma \operatorname{Der}(A)),
\end{gathered}
$$

where

$$
\mathbb{F}_{\Lambda \text { colie }^{+}}(A, \Sigma \operatorname{Der}(A))=\mathbb{F}_{\Lambda \text { colie }}(A) \oplus \mathbb{F}_{\Lambda \text { colie }^{+}}(A, \Sigma \operatorname{Der}(A))^{+}
$$

is the direct sum of the cofree $\Lambda$ colie-coalgebra $\mathbb{F}_{\Lambda \text { colie }}(A)$ cogenerated by $A$ and the cofree comodule $\mathbb{F}_{\Lambda \text { colie }}+(A, \Sigma \operatorname{Der}(A))^{+}$over $\mathbb{F}_{\Lambda \text { colie }}(A)$ cogenerated by $\operatorname{Der}(A)$ placed in degree 1 .

It is not hard to see that $\Xi(A)$ consists of sums of expressions:

$$
\left(\lambda_{1} \otimes p_{1}\right)^{S_{\left|p_{1}\right|} \ldots\left(\lambda_{k} \otimes p_{k}\right)^{S_{\left|p_{k}\right|}}}
$$

where $p_{i}$ are monomials in $\bigotimes(A \oplus \operatorname{Der}(A))$ in which an element of $\operatorname{Der}(A)$ can appear at most once, $\lambda_{i}$ is an element of $\Lambda$ colie $\left(\left|p_{i}\right|\right)$ and $\left|p_{i}\right|$ denotes the degree of $p_{i}$.

We conclude this section with the following theorem.

Theorem 3. The map $m$ (2.8) vanishes on monomials of $\Xi(A)$ which have more than two components. Furthermore,

$$
m\left(P_{1} P_{2}\right)=(-)^{\left|P_{1}\right|}\left[P_{1}, P_{2}\right]_{G}, \quad m\left\langle P_{1}, P_{2}\right\rangle=(-)^{\left|P_{1}\right|} P_{1} \cdot P_{2}
$$

whenever the monomial $P_{1} P_{2}$ (resp. the monomial $\left\langle P_{1}, P_{2}\right\rangle$ ) belongs to $\Xi(A)$. 
Proof. Equation (2.13) follows easily from the degree bookkeeping and it remains to prove that the map $m$ (2.8) vanishes on monomials of $\Xi(A)$ which have more than two components. The latter is equivalent to the following equations

$$
\begin{gathered}
m\left\langle v, f_{1}, f_{2}\right\rangle=0, \quad m\left\langle f_{1}, v, f_{2}\right\rangle=m\left\langle f_{1}, f_{2}, v\right\rangle=0, \\
m\left(\left\langle v_{1}, f\right\rangle v_{2}\right)=0, \\
m\left(v v_{1} v_{2}\right)=0,
\end{gathered}
$$

for all $v, v_{1}, v_{2} \in \operatorname{Der}(A)$ and $f, f_{1}, f_{2} \in A$, where we use the notation

$$
\left\langle P_{1}, \ldots, P_{m}\right\rangle, \quad P_{i} \in C^{\bullet}(A)
$$

for elements in $\mathbb{F}_{\text {colie }}\left(\Sigma^{-1} C^{\bullet}(A)\right)$ keeping in mind the realization (1.27).

Indeed, any other higher combination will have degree $<-1$ while the complex $C^{\bullet}(A)$ lies in non-negative degrees. Since the map (2.8) is of degree one all these combinations get sent to zero.

Equation (2.16) follows from Theorem 2 and the second pair of equations in (2.14) follows from the first equation. The latter can be proved using the fact that $m$ has to vanish on shuffle-products (1.28).

Thus we are left with the pair of the following equations:

$$
m\left\langle v, f_{1}, f_{2}\right\rangle=0, \quad m\left\langle v_{1}, f\right\rangle v_{2}=0 .
$$

Since the map $m$ (2.8) is of degree 1 and $\left\langle v, f_{1}, f_{2}\right\rangle$ is of degree -1 in the coalgebra

$$
\Sigma^{2} \mathbb{F}_{\text {cocomm }}\left(\Sigma^{-1} \mathbb{F}_{\text {colie }}\left(\Sigma^{-1} C^{\bullet}(A)\right)\right)
$$

we have that

$$
m\left\langle v, f_{1}, f_{2}\right\rangle \in C^{0}(A)=A .
$$

Thus, using the fact that the map $m$ (2.8) is given in terms of the brace operations [11], [18], we conclude that the most general expression for $m\left\langle v, f_{1}, f_{2}\right\rangle$ is

$$
m\left\langle v, f_{1}, f_{2}\right\rangle=\alpha f_{1} v\left(f_{2}\right)+\beta f_{2} v\left(f_{1}\right),
$$

where $\alpha, \beta \in \mathbb{K}$ and $v(\cdot)$ denotes the action of the derivation on a function.

On the other hand, applying the corestriction $u_{C^{\bullet}{ }_{(A)}}(2.7)$ to the equation

$$
M^{2}\left(\left\langle v, f_{1}, f_{2}, f_{3}\right\rangle\right)=0
$$

and using (2.13) we get

$$
f_{3} \cdot m\left\langle v, f_{1}, f_{2}\right\rangle-m\left\langle f_{1} \cdot v, f_{2}, f_{3}\right\rangle+m\left\langle v, f_{1} \cdot f_{2}, f_{3}\right\rangle-m\left\langle v, f_{1}, f_{2} \cdot f_{3}\right\rangle=0,
$$

where $\cdot$ denotes the cup product in $C^{\bullet}(A)$. 
Due to (2.18) equation (2.19) boils down to

$$
\beta f_{2} \cdot f_{3} \cdot v\left(f_{1}\right)-\alpha f_{1} \cdot f_{2} \cdot v\left(f_{3}\right)=0 .
$$

Since (2.20) holds for any associative algebra $A$ the coefficients $\alpha$ and $\beta$ vanish and the first equation in (2.17) is satisfied.

Similar degree bookkeeping shows that

$$
m\left(\left\langle v_{1}, f\right\rangle v_{2}\right) \in C^{0}(A)=A
$$

Hence, the most general expression for $m\left(\left\langle v_{1}, f\right\rangle v_{2}\right)$ is

$$
m\left(\left\langle v_{1}, f\right\rangle v_{2}\right)=\mu v_{2}\left(v_{1}(f)\right)+v v_{1}\left(v_{2}(f)\right),
$$

where $\mu, v \in \mathbb{K}$.

Applying the corestriction $u_{C^{\bullet}(A)}(2.7)$ to the equation

$$
M^{2}\left(\left\langle v_{1}, f\right\rangle v_{2} v_{3}\right)=0
$$

and using (2.13) we get

$$
v_{2}\left(m\left(\left\langle v_{1}, f\right\rangle v_{3}\right)\right)+m\left(\left\langle\left[v_{1}, v_{2}\right]_{G}, f\right\rangle v_{3}\right)+m\left(\left\langle v_{1}, v_{3}(f)\right\rangle v_{2}\right)-(2 \leftrightarrow 3)=0 .
$$

Due to (2.21) equation (2.22) boils down to

$$
\mu\left[v_{2}, v_{3}\right]_{G}\left(v_{1}(f)\right)+v v_{1}\left(\left[v_{2}, v_{3}\right]_{G}(f)\right)=0 .
$$

Since equation (2.23) holds for any associative algebra $A$ the coefficients $\mu$ and $v$ necessarily vanish and the second equation in (2.17) is satisfied.

The theorem is proved.

\section{The formality theorem}

In this section we construct a chain of quasi-isomorphisms between the homotopy Gerstenhaber algebra $C^{\bullet}(A)$ of Hochschild cochains of a regular commutative algebra $A$ and the Gerstenhaber algebra $V^{\bullet}(A)$ (2.9) of multiderivations of $A$.

First, we observe that, as in equation (1.15), the map (1.12) gives us a homotopy morphism

$$
\chi^{C^{\bullet}(A)}: C^{\bullet}(A) \succ \Omega_{\mathbf{e}_{2}}\left(\mathbb{F}_{\mathbf{e}_{2} \vee}\left(C^{\bullet}(A)\right), M\right),
$$

where $M$ is the codifferential of the coalgebra $\mathbb{F}_{\mathbf{e}_{2}} \vee\left(C^{\bullet}(A)\right)$ defining the homotopy Gerstenhaber algebra structure on $C^{\bullet}(A)$. Due to Proposition 3 the homotopy morphism (3.1) is a quasi-isomorphism. 
Second, Theorem 1 gives a quasi-isomorphism of DG Gerstenhaber algebras

$$
\eta_{\mathbf{e}_{2}}: \Omega_{\mathbf{e}_{2}}\left(B_{\mathbf{e}_{2} \vee}\left(V^{\bullet}(A)\right)\right) \rightarrow V^{\bullet}(A),
$$

where $V^{\bullet}(A)$ is equipped with the vanishing differential.

Thus it suffices to connect the DG Gerstenhaber algebras

$$
\Omega_{\mathbf{e}_{2}}\left(B_{\mathbf{e}_{2} \vee}\left(V^{\bullet}(A)\right)\right)
$$

and

$$
\Omega_{\mathbf{e}_{2}}\left(\mathbb{F}_{\mathbf{e}_{2}} \vee\left(C^{\bullet}(A)\right), M\right)
$$

by a chain of quasi-isomorphisms. For this purpose we will use the sub $\mathbf{e}_{\mathbf{2}}{ }^{\vee}$-coalgebra

$$
\Xi(A)=\mathbb{F}_{\Lambda^{2} \text { cocomm }}\left(\mathbb{F}_{\Lambda \text { colie }^{+}}+(A, \Sigma \operatorname{Der}(A))\right)
$$

of $\mathbb{F}_{\mathbf{e}_{2}} \vee\left(C^{\bullet}(A)\right)$ introduced in equation (2.11).

Notice that $\Xi(A)$ is also a sub $\mathbf{e}_{2} \vee$-coalgebra of $\mathbb{F}_{\mathbf{e}_{2}} \vee\left(V^{\bullet}(A)\right)$. On the other hand, due to Remark 1 after Theorem 1

$$
B_{\mathbf{e}_{2}} \vee\left(V^{\bullet}(A)\right)=\mathbb{F}_{\mathbf{e}_{2} \vee}\left(V^{\bullet}(A)\right)
$$

as $\mathbf{e}_{2}{ }^{\vee}$-coalgebras. Thus the natural question arises of whether $\Xi(A) \subset \mathbb{F}_{\mathbf{e}_{2} \vee}\left(V^{\bullet}(A)\right)$ is stable under the codifferential

$$
d_{V^{\bullet}(A)}: \mathbb{F}_{\mathbf{e}_{2} \vee}\left(V^{\bullet}(A)\right) \rightarrow \mathbb{F}_{\mathbf{e}_{2} \vee}\left(V^{\bullet}(A)\right)
$$

of $B_{\mathbf{e}_{2}} \vee\left(V^{\bullet}(A)\right)$.

The following proposition gives the positive answer to this question.

Proposition 7. Let $_{V^{\bullet}(A)}$ be the codifferential of the DG $\mathbf{e}_{2}{ }^{\vee}$-coalgebra $B_{\mathbf{e}_{2}}{ }^{\vee}\left(V^{\bullet}(A)\right)$. Then

$$
d_{V^{\bullet}(A)} \mid \Xi(A) \subset \Xi(A)
$$

Proof. According to the construction (see p. 26 in [12]) of the functor $B_{\mathcal{O}^{\vee}}$ of the adjunction (1.10) the proposition follows easily from the obvious inclusions

$$
\begin{gathered}
A \cdot \operatorname{Der}(A) \subset \operatorname{Der}(A), \\
{[\operatorname{Der}(A), A]_{\mathrm{SN}} \subset A, \quad[\operatorname{Der}(A), \operatorname{Der}(A)]_{\mathrm{SN}} \subset \operatorname{Der}(A),}
\end{gathered}
$$

where $\cdot$ and $[,]_{\mathrm{SN}}$ denote the product and the Lie bracket in $V^{\bullet}(A)$, respectively.

We denote the embedding of $\Xi(A)$ (2.11) into the DG $\mathbf{e}_{2}{ }^{\vee}$-coalgebra $B_{\mathbf{e}_{2}} \vee\left(V^{\bullet}(A)\right)$ by $\iota$,

$$
\iota: \Xi(A) \hookrightarrow B_{\mathbf{e}_{2}} \vee\left(V^{\bullet}(A)\right) .
$$


We will also use the same notation $d_{V^{\bullet}(A)}$ for the restriction of the codifferential (3.3) to $\Xi(A)$.

Similarly to Proposition 7, Theorem 3 shows that the natural embedding of $\mathbf{e}_{2}{ }^{\vee}$ coalgebras

$$
\sigma: \Xi(A) \hookrightarrow \mathbb{F}_{\mathbf{e}_{2} \vee}\left(C^{\bullet}(A)\right)
$$

is also compatible with the codifferentials $d_{V^{\bullet}(A)}$ and $M$, where the codifferential $M$ on $\mathbb{F}_{\mathbf{e}_{2} \vee}\left(C^{\bullet}(A)\right)$ is the one corresponding to the homotopy Gerstenhaber algebra structure on $C^{\bullet}(A)$.

Thus we get the following pair of morphisms of DG $\mathbf{e}_{2}{ }^{\vee}$-coalgebras:

$$
\left(\mathbb{F}_{\mathbf{e}_{2} \vee}\left(C^{\bullet}(A)\right), M\right) \stackrel{\sigma}{\longleftarrow}\left(\Xi(A), d_{V^{\bullet}(A)}\right) \stackrel{\iota}{\longrightarrow} B_{\mathbf{e}_{2} \vee}\left(V^{\bullet}(A)\right)
$$

Applying to (3.6) the functor $\Omega_{\mathbf{e}_{2}}$ (1.10) we get the pair of morphisms of DG Gerstenhaber algebras:

$$
\Omega_{\mathbf{e}_{2}}\left(\mathbb{F}_{\mathbf{e}_{2} \vee}\left(C^{\bullet}(A)\right), M\right) \stackrel{\Omega_{\mathrm{e}_{2}}(\sigma)}{\longleftarrow} \Omega_{\mathbf{e}_{2}}\left(\Xi(A), d_{V^{\bullet}(A)}\right) \stackrel{\Omega_{\mathbf{e}_{2}}(t)}{\longrightarrow} \Omega_{\mathbf{e}_{2}}\left(B_{\mathbf{e}_{2}} \vee\left(V^{\bullet}(A)\right)\right) .
$$

Combining this pair with (3.1) and (3.2) we get the following diagram of (homotopy) morphisms of (homotopy) Gerstenhaber algebras

$$
\begin{aligned}
& C^{\bullet}(A) \stackrel{\chi^{C^{\bullet}(A)}}{\longrightarrow} \Omega_{\mathbf{e}_{2}}\left(\mathbb{F}_{\mathbf{e}_{2} \vee}\left(C^{\bullet}(A)\right), M\right) \\
& \begin{array}{c}
\uparrow \Omega_{\mathrm{e}_{2}}(\sigma) \\
\Omega_{\mathrm{e}_{2}}\left(\Xi(A), d_{V^{\bullet}(A)}\right)
\end{array} \\
& V^{\bullet}(A)<\eta_{\mathbf{e}_{2}} \Omega_{\mathbf{e}_{2}}\left(B_{\mathbf{e}_{2} \vee}\left(V^{\bullet}(A)\right)\right)
\end{aligned}
$$

where $d_{V^{\bullet}(A)}$ is the restriction of the codifferential (3.3) to $\Xi(A)$.

Notice that in diagram (3.8) $\Omega_{\mathbf{e}_{2}}(\sigma), \Omega_{\mathbf{e}_{2}}(\iota)$, and $\eta_{\mathbf{e}_{2}}(1.11)$ are honest morphisms of DG Gerstenhaber algebras and $\chi^{C^{\bullet}(A)}$ is a homotopy morphism.

The main result of this paper can be formulated as follows:

Theorem 4. Let $A$ be a regular commutative (associative) algebra (with unit) over a field $\mathbb{K}$ of characteristic zero. If $C^{\bullet}(A)$ is the normalized Hochschild cochain complex of $A$,

$$
M: \mathbb{F}_{\mathbf{e}_{2} \vee}\left(C^{\bullet}(A)\right) \rightarrow \mathbb{F}_{\mathbf{e}_{2} \vee}\left(C^{\bullet}(A)\right)
$$

is the homotopy Gerstenhaber algebra structure on $C^{\bullet}(A)$ induced by the action of the little disk operad, $\Xi(A)$ is the $\mathbf{e}_{2}{ }^{\vee}$-coalgebra introduced in (2.11), $\Omega$ and $B$ are 
the adjoint functors (1.10), and $\iota, \sigma$ are embeddings defined in (3.4) and (3.5) then (3.8) is a chain of quasi-isomorphisms of (homotopy) Gerstenhaber algebras.

Proof. The map $\eta_{\mathbf{e}_{2}}$ in (3.8) is a quasi-isomorphism by Theorem 1 and $\chi^{C^{\bullet}(A)}$ in (3.8) is a quasi-isomorphism by Proposition 3. To prove that $\Omega_{\mathbf{e}_{2}}(\iota)$ is a quasi-isomorphism we show that so is the composition $\eta_{\mathbf{e}_{2}} \circ \Omega_{\mathbf{e}_{2}}(\iota)$. Then the fact that $\Omega_{\mathbf{e}_{2}}(\sigma)$ is a quasi-isomorphism will follow from the Hochschild-Kostant-Rosenberg theorem for $C^{\bullet}(A)[17]$.

Let us consider the composition

$$
v=\eta_{\mathbf{e}_{2}} \circ \Omega_{\mathbf{e}_{2}}(\iota): \Omega_{\mathbf{e}_{2}}\left(\Xi(A), d_{V^{\bullet}(A)}\right) \rightarrow V^{\bullet}(A) .
$$

This is a map of DG Gerstenhaber algebras where the algebra $V^{\bullet}(A)(2.9)$ carries the vanishing differential.

Since $\operatorname{Der}(A)$ is a module over $A$ we may regard the pair $(A, \Sigma \operatorname{Der}(A))$ as a comm $^{+}$-algebra. Therefore, due to equation 1.29 , the functor $B_{\Lambda \text { colie }}+$ of the adjunction (1.10) gives us the pair

$$
\left(B_{\Lambda \text { colie }}(A), B_{\Lambda \text { colie }^{+}}(A, \Sigma \operatorname{Der}(A))^{+}\right)
$$

of the DG $\Lambda$ colie coalgebra $B_{\Lambda \text { colie }}(A)$ and its DG comodule $B_{\Lambda \text { colie }}+(A, \Sigma \operatorname{Der}(A))^{+}$. Notice that as a $\Lambda$ colie comodule $\left.B_{\Lambda \text { colie }}+(A, \Sigma \operatorname{Der}(A))^{+}\right)$is a cofree comodule over $B_{\Lambda \text { colie }}(A)$ cogenerated by $\operatorname{Der}(A)$ placed in degree 1 .

It is convenient to denote by $B_{\Lambda \text { colie }}+(A, \Sigma \operatorname{Der}(A))$ the direct sum

$$
B_{\Lambda \text { colie }^{+}}(A, \Sigma \operatorname{Der}(A))=B_{\Lambda \text { colie }}(A) \oplus B_{\Lambda \text { colie }^{+}}(A, \Sigma \operatorname{Der}(A))^{+} .
$$

It is easy to see that (3.12) is naturally a DG $\Lambda$ colie-coalgebra. Furthermore, since the direct sum $A \oplus \operatorname{Der}(A)$ carries a Lie bracket which is compatible with the product on $A$ and the $A$-module structure on $\operatorname{Der}(A)$, the graded vector space $B_{\Lambda \text { colie }}+(A, \Sigma \operatorname{Der}(A))$ is equipped ${ }^{5}$ with $\Lambda$ lie-algebra structure which is compatible with the DG $\Lambda$ coliecoalgebra. Due to this fact we can apply to $B_{\Lambda \text { colie }}+(A, \Sigma \operatorname{Der}(A))$ the functor $B_{\Lambda^{2}}$ cocomm and get the following DG $\mathbf{e}_{2}{ }^{\vee}$-coalgebra

$$
B_{\Lambda^{2} \text { cocomm }}\left(B_{\Lambda \text { colie }^{+}}(A, \Sigma \operatorname{Der}(A))\right) .
$$

It is not hard to see that

$$
\left(\Xi(A), d_{V^{\bullet}(A)}\right) \cong B_{\Lambda^{2} \text { cocomm }}\left(B_{\Lambda \operatorname{colie}^{+}}(A, \Sigma \operatorname{Der}(A))\right)
$$

as DG $\mathbf{e}_{2}{ }^{\vee}$ coalgebras, where $d_{V^{\bullet}(A)}$ is, as above, the restriction of the codifferential of $B_{\mathbf{e}_{2}} \vee\left(V^{\bullet}(A)\right)$ to $\Xi(A)$.

\footnotetext{
${ }^{5}$ See Subsection 1.2.2 in which we give a detailed explanation of how the functor $B_{\Lambda \text { colie }}$ can be extended to a functor from the category of Gerstenhaber algebras to the category of DG Lie bialgebras with Lie brackets of degree -1 and Lie cobrackets of degree 1 .
} 
Thus our purpose is to prove that the map $v(3.10)$,

$$
v: \Omega_{\text {comm }} \Omega_{\Lambda \text { lie }} B_{\Lambda^{2} \text { cocomm }} B_{\Lambda \text { colie }^{+}}(A, \Sigma \operatorname{Der}(A)) \rightarrow V^{\bullet}(A),
$$

is a quasi-isomorphism of DG Gerstenhaber algebras.

To do this we notice that applying the functor $\Omega_{\text {comm }}$ to the DG $\Lambda$ colie-coalgebra $B_{\Lambda \text { colie }}+(A, \Sigma \operatorname{Der}(A))(3.12)$ we get the following DG Gerstenhaber algebra

$$
\Omega_{\text {comm }}\left(B_{\Lambda \text { colie }^{+}}(A, \Sigma \operatorname{Der}(A))\right)
$$

with the obvious commutative product and the Lie bracket induced from $B_{\Lambda \text { colie }}+(A, \Sigma \operatorname{Der}(A))$.

Furthermore, there is canonical map (of DG Gerstenhaber algebras)

$$
\begin{aligned}
v_{1}: \Omega_{\text {comm }} \Omega_{\Lambda \text { lie }} B_{\Lambda^{2} \text { cocomm }} & B_{\Lambda \text { colie }^{+}}(A, \Sigma \operatorname{Der}(A)) \\
& \rightarrow \Omega_{\text {comm }} B_{\Lambda \text { colie }^{+}}(A, \Sigma \operatorname{Der}(A)),
\end{aligned}
$$

which is obtained by applying the functor $\Omega_{\text {comm }}$ to the counit (1.11)

$$
\eta_{\Lambda \text { lie }}: \Omega_{\Lambda \text { lie }} B_{\Lambda^{2} \text { cocomm }} B_{\Lambda \text { colie }^{+}}(A, \Sigma \operatorname{Der}(A)) \rightarrow B_{\Lambda \text { colie }^{+}}(A, \Sigma \operatorname{Der}(A)) \text {. }
$$

On the other hand we can define the following map of (DG) Gerstenhaber algebras

$$
v_{2}: \Omega_{\text {comm }} B_{\Lambda \text { colie }^{+}}+(A, \operatorname{Der}(A)) \rightarrow V^{\bullet}(A) .
$$

As a map of commutative algebras $\nu_{2}$ is defined by its restriction to the space $B_{\Lambda \text { colie }}+(A, \operatorname{Der}(A))$ of generators of $\Omega_{\text {comm }} B_{\Lambda \text { colie }}+(A, \operatorname{Der}(A))$. Namely, we define $v_{2}$ by setting

$$
\left.v_{2}\right|_{B_{\Lambda \text { colie }}+}(A, \operatorname{Der}(A))=i \circ u_{A \oplus \Sigma \operatorname{Der}(A)},
$$

where $u_{A \oplus \Sigma \operatorname{Der}(A)}$ is the corestriction (1.1) from $B_{\Lambda \text { colie }}+(A, \operatorname{Der}(A))$ to the space of its cogenerators $A \oplus \Sigma \operatorname{Der}(A)$ and $i$ is the natural embedding 6

$$
i: A \oplus \Sigma \operatorname{Der}(A) \hookrightarrow V^{\bullet}(A) .
$$

It is not hard to check that the map $v_{2}$ (3.16) defined by (3.17) is compatible both with the differentials and the Lie brackets.

Next, one can show that the map $v$ (3.14) is the composition

$$
v=v_{2} \circ v_{1}
$$

of the maps (3.15) and (3.16).

\footnotetext{
${ }^{6}$ Recall that $V^{\bullet}(A)=S_{A}(\Sigma \operatorname{Der}(A))$ (2.9).
} 
In order to prove that $v_{1}(3.15)$ is a quasi-isomorphism we introduce the following increasing filtration on the space $B_{\Lambda \text { colie }}+(A, \Sigma \operatorname{Der}(A))$

$$
\begin{gathered}
F^{0} B_{\Lambda \text { colie }^{+}}(A, \Sigma \operatorname{Der}(A)) \subset \cdots \subset F^{m} B_{\Lambda \text { colie }^{+}}(A, \Sigma \operatorname{Der}(A)) \\
\subset F^{m+1} B_{\Lambda \text { colie }^{+}}(A, \Sigma \operatorname{Der}(A)) \subset \cdots \subset B_{\Lambda \text { colie }^{+}}(A, \Sigma \operatorname{Der}(A)),
\end{gathered}
$$

where the subspace $F^{m} B_{\Lambda \text { colie }}+(A, \Sigma \operatorname{Der}(A))$ is spanned by vectors

$$
(\lambda \otimes p)^{S_{n}}, \quad n \leq m+1,
$$

where $\lambda \in \Lambda \operatorname{colie}(n)$ and $p$ is a degree $n$ monomial in $\otimes(A \oplus \operatorname{Der}(A))$ in which an element of $\operatorname{Der}(A)$ can appear at most once. This filtration is obviously compatible with the algebraic structures on $B_{\Lambda \text { colie }}+(A, \Sigma \operatorname{Der}(A))$. Furthermore, both the codifferential and the cobracket on $B_{\Lambda \text { colie }^{+}}(A, \Sigma \operatorname{Der}(A))$ lower the filtration by 1 . Hence, on the associated graded space $\operatorname{Gr}\left(B_{\Lambda \text { colie }}+(A, \Sigma \operatorname{Der}(A))\right)$ only the structure of a $\Lambda$ lie-algebra survives, while the cobracket and the codifferential vanish.

The filtration (3.19) canonically extends to exhausting increasing filtrations on the complexes

$$
\Omega_{\text {comm }} \Omega_{\Lambda \text { lie }} B_{\Lambda^{2} \text { cocomm }} B_{\Lambda \text { colie }^{+}}(A, \Sigma \operatorname{Der}(A))
$$

and

$$
\Omega_{\text {comm }} B_{\Lambda \text { colie }^{+}}(A, \Sigma \operatorname{Der}(A)) .
$$

The map $v_{1}(3.15)$ is obviously compatible with the resulting filtrations. Moreover, since the cobracket in $B_{\Lambda \text { colie }}+(A, \Sigma \operatorname{Der}(A))$ lowers the filtration by 1 the associated graded complexes are

$$
\mathbb{F}_{\text {comm }} \Omega_{\Lambda \text { lie }} B_{\Lambda^{2} \text { cocomm }} \operatorname{Gr}\left(B_{\Lambda \text { colie }^{+}}(A, \Sigma \operatorname{Der}(A))\right)
$$

and

$$
\mathbb{F}_{\text {comm }} \operatorname{Gr}\left(B_{\Lambda \text { colie }^{+}}+(A, \Sigma \operatorname{Der}(A))\right),
$$

respectively, and the associated graded morphism

$$
\operatorname{Gr}\left(v_{1}\right)=\mathbb{F}_{\text {comm }}\left(\eta_{\Lambda \text { lie }}\right),
$$

where

$\eta_{\Lambda \text { lie }}: \Omega_{\Lambda \text { lie }} B_{\Lambda^{2} \text { cocomm }} \operatorname{Gr}\left(B_{\Lambda \text { colie }}+(A, \Sigma \operatorname{Der}(A))\right) \rightarrow \operatorname{Gr}\left(B_{\Lambda \text { colie }}+(A, \Sigma \operatorname{Der}(A))\right)$, is the counit (1.11) of the adjunction (1.10), and $\operatorname{Gr}\left(B_{\Lambda \text { colie }^{+}}(A, \Sigma \operatorname{Der}(A))\right)$ is viewed as the $\Lambda$ lie-algebra.

By Theorem $1, \eta_{\Lambda \text { lie }}$ is a quasi-isomorphism. On the other hand, the functor $\mathbb{F}_{\text {comm }}$ is exact since the underlying field $\mathbb{K}$ has characteristic zero. Thus the map $v_{1}(3.15)$ is indeed a quasi-isomorphism. 
Let us prove that the map $v_{2}$ (3.16) is a quasi-isomorphism.

Due to Theorem 1 and Proposition 6 we have the quasi-isomorphism

$$
\eta_{\text {comm }}: \Omega_{\text {comm }}\left(B_{\Lambda \text { colie }}(A)\right) \rightarrow A
$$

of DG commutative algebras (with $A$ carrying the vanishing differential) and the quasi-isomorphism

$$
\eta_{\text {comm }^{+}}: \Omega_{\text {comm }}\left(B_{\Lambda \text { colie }}(A)\right) \otimes B_{\Lambda \text { colie }^{+}}(A, \Sigma \operatorname{Der}(A))^{+} \rightarrow \Sigma \operatorname{Der}(A)
$$

of the corresponding (DG) modules over the (DG) commutative algebras $A$ and $\Omega_{\text {comm }}\left(B_{\Lambda \text { colie }}(A)\right)$.

Since $A$ is regular $\operatorname{Der}(A)$ is a flat module over $A$. Hence the maps (3.20) and (3.21) induce the following quasi-isomorphism of DG commutative algebras

$$
\begin{aligned}
\hat{\eta}: S_{\Omega_{\text {comm }}\left(B_{\Lambda \text { colie }}(A)\right)}\left(\Omega_{\text {comm }}\left(B_{\Lambda \text { colie }}(A)\right)\right. & \left.\otimes B_{\Lambda \text { colie }}(A, \Sigma \operatorname{Der}(A))^{+}\right) \\
& \rightarrow S_{A}(\Sigma \operatorname{Der}(A))
\end{aligned}
$$

On the other hand, it is obvious that the DG commutative algebra

$$
S_{\Omega_{\text {comm }}\left(B_{\Lambda \text { colie }}(A)\right)}\left(\Omega_{\text {comm }}\left(B_{\Lambda \text { colie }}(A)\right) \otimes B_{\Lambda \text { colie }^{+}}(A, \Sigma \operatorname{Der}(A))^{+}\right)
$$

is isomorphic to

$$
\Omega_{\text {comm }}\left(B_{\Lambda \text { colie }^{+}}(A, \Sigma \operatorname{Der}(A))\right),
$$

where the DG $\Lambda$ colie-coalgebra $B_{\Lambda \text { colie }}+(A, \Sigma \operatorname{Der}(A))$ is defined in equation (3.12).

Thus $\hat{\eta}$ (3.22) is in fact a quasi-isomorphism

$$
\hat{\eta}: \Omega_{\text {comm }}\left(B_{\Lambda \text { colie }^{+}}(A, \Sigma \operatorname{Der}(A))\right) \rightarrow S_{A}(\Sigma \operatorname{Der}(A)) .
$$

It is not hard to see that $\hat{\eta}$ coincides with the map $v_{2}$ (3.16). Hence, $v_{2}$ is a quasiisomorphism.

Due to equation (3.18) the map $v(3.10)$ is a quasi-isomorphism. Hence, so is $\Omega_{\mathrm{e}_{2}}(\iota)$.

It remains to prove that $\Omega_{\mathbf{e}_{2}}(\sigma)$ in (3.8) is a quasi-isomorphism. To prove this fact we notice that due to the regularity of $A$ the cohomology of $C^{\bullet}(A)$ is generated by classes of $A \subset C^{\bullet}(A)$ and $\operatorname{Der}(A) \subset C^{\bullet}(A)$. The restriction of $\Omega_{\mathbf{e}_{2}}(\sigma)$ to these representatives in $\Omega_{\mathbf{e}_{2}}\left(\Xi(A), d_{V^{\bullet}(A)}\right)$ and $\Omega_{\mathbf{e}_{2}}\left(\mathbb{F}_{\mathbf{e}_{2} \vee}\left(C^{\bullet}(A)\right), M\right)$ gives the identity map. Hence, $\Omega_{\mathbf{e}_{2}}(\sigma)$ is a quasi-isomorphism and the theorem is proved.

\section{Generalizations and applications}

One can notice that the proof of Theorem 4 is based on the flatness of the module $\operatorname{Der}(A)$ over $A$ and the fact that the cohomology of $C^{\bullet}(A)$ is generated by classes of $A \subset C^{\bullet}(A)$ and $\operatorname{Der}(A) \subset C^{\bullet}(A)$. 
This observation allows us to reformulate the proof of Theorem 4 and get the following result:

Theorem 5. If $\mathcal{M}$ is a smooth real manifold and $D^{\bullet}(\mathcal{M})$ is the graded vector space of polydifferential operators on $\mathcal{M}$ then the homotopy Gerstenhaber algebra on $D^{\bullet}(\mathcal{M})$ induced by the map (2.1) is formal.

Let $X$ be a smooth complex manifold (resp. a smooth algebraic variety over a field $\mathbb{K}$ of characteristic zero) and $D_{X}^{\cdot}$ be the sheaf of holomorphic (resp. algebraic) polydifferential operators on $X$. Due to the map (2.1) $D_{X}^{\circ}$ is a sheaf of homotopy Gerstenhaber algebras. Using the argument similar to the argument [29] for smooth real manifold it is not hard to show that the sheaf of cohomology of $D_{X}^{*}$ is the exterior algebra $\bigwedge^{\bullet} T_{X}$ of the tangent sheaf $T_{X}$. The product in $\bigwedge^{\bullet} T_{X}$ is the ordinary exterior product and the bracket is the Schouten-Nijenhuis bracket (see equation (3.20) on page 50 in [5]).

Replacing $A$ by $\mathcal{O}_{X}$, $\operatorname{Der}(A)$ by $T_{X}$, and $C^{\bullet}(A)$ by $D_{X}^{\bullet}$ in diagram (3.8) we get a chain of homotopy morphisms between sheaves of (homotopy) Gerstenhaber algebras connecting the sheaves $D_{X}^{\bullet}$ and $\bigwedge^{\bullet} T_{X}$. Generalizing the proof of Theorem 4 to this chain of morphisms we get the following theorem.

Theorem 6. The sheaf $D_{X}^{\bullet}$ of homotopy Gerstenhaber algebras is homotopy equivalent to the sheaf of its cohomology $\wedge^{\bullet} T_{X}$.

Let us apply this theorem and prove the last claim in paper [20]:

Corollary 2 (M. Kontsevich, [20], claim 8.4). Let $X$ be either a smooth complex manifold or a smooth algebraic variety (over a field $\mathbb{K}$ of characteristic zero). Let $\wedge^{\bullet} T_{X}$ be the exterior algebra of the tangent sheaf $T_{X}$ and $\Delta$ be the diagonal in $X \times X$. Then the graded commutative algebras

$$
H^{\bullet}\left(X, \bigwedge^{\bullet} T_{X}\right)
$$

and

$$
\operatorname{Ext}_{X \times X}^{\bullet}\left(\mathcal{O}_{\Delta}, \mathcal{O}_{\Delta}\right)
$$

are isomorphic.

Proof. First, due to Theorem 0.3 in [31] the ring $\operatorname{Ext}_{X \times X}^{\bullet}\left(\mathcal{O}_{\Delta}, \mathcal{O}_{\Delta}\right)$ is isomorphic to the hypercohomology

$$
\mathfrak{H}^{\bullet}\left(D_{X}^{\bullet}\right)
$$

of the sheaf $D_{X}^{\bullet}$ of polydifferential operators on $X$. The product on (4.1) is induced by the ordinary cup-product of polydifferential operators

$$
P_{1} \cup P_{2}\left(a_{1}, \ldots, a_{k_{1}+k_{2}}\right)=P\left(a_{1}, \ldots, a_{k_{1}}\right) Q\left(a_{k_{1}+1}, \ldots, a_{k_{1}+k_{2}}\right),
$$


where $P_{1}$ (resp. $P_{2}$ ) is a polydifferential operator of degree $k_{1}$ (resp. $k_{2}$ ).

The degree zero binary operation of the homotopy Gerstenhaber algebra on $D_{X}^{\bullet}$ induces another commutative product on the graded vector space (4.1). Let us denote this product by $\cup^{\prime}$.

By Theorem 6 the rings

$$
\left(\mathbb{W}^{\bullet}\left(D_{X}^{\bullet}\right), \cup^{\prime}\right)
$$

and

$$
H^{\bullet}\left(X, \bigwedge^{\bullet} T_{X}\right)
$$

are isomorphic. Therefore, it suffices to prove that $U^{\prime}$ coincides with the product induced by (4.2) on (4.1).

It is clear from the construction [23] of J. E. McClure and J. H. Smith that the degree zero binary operation - of the homotopy Gerstenhaber algebra on $D_{X}^{\bullet}$ is homotopic to the cup-product (4.2). In other words, for every pair of polydifferential operators $P_{1}$ and $P_{2}$

$$
P_{1} \cdot P_{2}=P_{1} \cup P_{2}+\partial \Psi\left(P_{1}, P_{2}\right)+\Psi\left(\partial P_{1}, P_{2}\right)+(-1)^{\left|P_{1}\right|} \Psi\left(P_{1}, \partial P_{2}\right),
$$

where $\partial$ is the Hochschild coboundary operator, $\left|P_{1}\right|$ stands for the degree of $P_{1}$ and $\Psi$ is a binary operation of degree -1 .

Let $\left(\check{C}^{\bullet}\left(D_{X}^{\bullet}\right), \check{\partial}+\partial\right)$ be the Čech complex of the sheaf $D_{X}^{\bullet}$. Let $P$ and $Q$ be cocycles in $\check{C}^{\bullet}\left(D_{X}^{\bullet}\right)$ representing cohomology classes $[P]$ and $[Q]$ in (4.1).

Equation (4.3) implies that

$$
P \cdot Q=P \cup Q+\partial \Psi(P, Q)+\Psi(\partial P, Q)+(-1)^{|P|} \Psi(P, \partial Q) .
$$

On the other hand $P$ and $Q$ are cocycles with respect to the differential $\check{\partial}+\partial$. Hence,

$$
P \cdot Q=P \cup Q+\partial \Psi(P, Q)-\Psi(\check{\partial} P, Q)-(-1)^{|P|} \Psi(P, \check{\partial} Q)
$$

or equivalently

$$
P \cdot Q=P \cup Q+(\partial+\check{\partial}) \Psi(P, Q) \text {. }
$$

Thus the product $U^{\prime}$ coincides with the product induced by (4.2) on (4.1) and the corollary follows.

Remark. Notice that (4.2) equips the sheaf $D_{X}^{\bullet}$ with a structure of a sheaf of DG associative algebras. In particular, the Čech complex $\check{C}^{\bullet}\left(D_{X}^{\bullet}\right)$ is naturally a DGA. It is not hard to prove that the graded algebra of cohomology of $\check{C}^{\bullet}\left(D_{X}^{\bullet}\right)$ is the graded (commutative) algebra $H^{\bullet}\left(X, \wedge^{\bullet} T_{X}\right)$. However, the question of whether $\check{C}^{\bullet}\left(D_{X}^{\bullet}\right)$ is quasi-isomorphic to its cohomology is much more subtle [19]. As far as we know the answer to this question is not yet found. 


\section{Concluding remarks}

We would like to mention papers [3] and [22] in which a super version of Kontsevich's formality theorem was applied to quantum reduction. In both of these papers the authors found an obstruction (a quantum anomaly in the terminology of [22]) to quantization of the quotient space. Notice that the proof of Theorem 4 admits an obvious generalization to the case of a graded ring $A$. In particular, one can easily extend a super version of Kontsevich's formality quasi-isomorphism constructed in [3] to a quasi-isomorphism of the corresponding homotopy Gerstenhaber algebras. It would be interesting to analyze the obstruction of A. S. Cattaneo, G. Felder, S. L. Lyakhovich, and A. A. Sharapov using the chain of quasi-isomorphisms (3.8).

We hope that the chain of quasi-isomorphisms (3.8) could be used to prove a part of Caldararu's conjecture formulated ${ }^{7}$ in [2].

We would like to mention that the zeroth part $C^{0}(A)=A$ of the Hochschild complex is essentially used in the proof of the main result. In particular, $\Xi(A)$ is the subalgebra of $\mathbb{F}_{\mathbf{e}_{2} \vee}\left(C^{\bullet}(A)\right)$ but not of $\mathbb{F}_{\mathbf{e}_{2} \vee}\left(C^{\geq 1}(A)\right)$, where $C^{\geq 1}(A)$ denotes the truncated Hochschild complex. It would be interesting to analyze the question of whether the truncated Hochschild complex is formal.

\section{References}

[1] J. M. Boardman and R. M. Vogt, Homotopy invariant algebraic structures on topological spaces. Lecture Notes in Math. 347, Springer-Verlag, Berlin 1973. Zbl 0285.55012 MR 0420609

[2] A. Căldăraru, The Mukai pairing, II: the Hochschild-Kostant-Rosenberg isomorphism. Adv. Math. 194 (2005), 34-66. Zbl 02181980 MR 2141853

[3] A. S. Cattaneo and G. Felder, Relative formality theorem and quantisation of coisotropic submanifolds. arXiv:math.QA/0501540

[4] A. V. Dolgushev, Covariant and equivariant formality theorems. Adv. Math. 191 (2005), 147-177. Zbl 02134411 MR 2102846

[5] A. V. Dolgushev, A proof of Tsygan's formality conjecture for an arbitrary smooth manifold. PhD thesis, M.I.T. arXiv:math.QA/0504420

[6] B. V. Fedosov, A simple geometrical construction of deformation quantization. J. Differential Geom. 40 (1994), 213-238. Zbl 0812.53034 MR 1293654

[7] B. Fresse, Koszul duality of operads and homology of partition posets. In Homotopy theory: relations with algebraic geometry, group cohomology, and algebraic $K$-theory (Evanston, 2002), Contemp. Math. 346, Amer. Math. Soc. Providence, RI, 2004, 115-215. Zbl 1077.18007 MR 2066499

\footnotetext{
${ }^{7}$ See Conjecture 5.2 in [2].
} 
[8] I. M. Gel'fand and D. B. Fuks, Cohomology of the Lie algebra of formal vector fields. Izv. Akad. Nauk SSSR Ser. Mat. 34 (1970), 322-337; English transl. Math. USSR-Izv. 4 (1970), 327-342. Zbl 0216.20302 MR 0266195

[9] I. M. Gel'fand and D. A. Každan, Some problems of differential geometry and the calculation of cohomologies of Lie algebras of vector fields. Dokl. Akad. Nauk SSSR 200 (1971), 269-272; English transl. Soviet Math. Dokl. 12 (1971), 1367-1370. Zbl 0238.58001 MR 0287566

[10] M. Gerstenhaber, The cohomology structure of an associative ring. Ann. of Math. (2) 78 (1963), 267-288. Zbl 0131.27302 MR 0161898

[11] E. Getzler, Cartan homotopy formulas and the Gauss-Manin connection in cyclic homology. In Quantum deformations of algebras and their representations, Israel Math. Conf. Proc. 7, Bar-Ilan Univ., Ramat Gan, 1993, 65-78. Zbl 0844.18007 MR 1261901

[12] E. Getzler and J. D. S. Jones, Operads, homotopy algebra and iterated integrals for double loop spaces. arXiv:hep-th/9403055

[13] V. Ginzburg and M. Kapranov, Koszul duality for operads. Duke Math. J. 76 (1994), 203-272. Zbl 0855.18006 MR 1301191

[14] G. Halbout, Globalization of Tamarkin's formality theorem. Lett. Math. Phys. 71 (2005), 39-48. Zbl 1081.53079 MR 2136736

[15] V. Hinich, Homological algebra of homotopy algebras. Comm. Algebra 25 (1997), 3291-3323. Zbl 0894.18008 MR 1465117

[16] V. Hinich, Tamarkin's proof of Kontsevich formality theorem. Forum Math. 15 (2003), 591-614. Zbl 1081.16014 MR 1978336

[17] G. Hochschild, B. Kostant, and A. Rosenberg, Differential forms on regular affine algebras. Trans. Amer. Math. Soc. 102 (1962), 383-408. Zbl 0102.27701 MR 0142598

[18] T. V. Kadeishvili, The structure of the $A(\infty)$-algebra, and the Hochschild and Harrison cohomologies. Trudy Tbiliss. Mat. Inst. Razmadze Akad. Nauk Gruzin. SSR 91 (1988), 19-27 (in Russian). Zbl 0717.55011 MR 1029003

[19] M. Kontsevich, Operads and motives in deformation quantization. Lett. Math. Phys. 48 (1999), 35-72. Zbl 0945.18008 MR 1718044

[20] M. Kontsevich, Deformation quantization of Poisson manifolds. Lett. Math. Phys. 66 (2003), 157-216. Zbl 1058.53065 MR 2062626

[21] M. Kontsevich and Y. Soibelman, Deformations of algebras over operads and Deligne's conjecture. In Conférence Moshé Flato 1999, Vol. I (Dijon), Math. Phys. Stud. 21, Kluwer Acad. Publ., Dordrecht 2000, 255-307. Zbl 0972.18005 MR 1805894

[22] S. L. Lyakhovich and A. A. Sharapov, BRST theory without Hamiltonian and Lagrangian. J. High Energy Phys. 03 (2005), 011. MR 2151981

[23] J. E. McClure and J. H. Smith, A solution of Deligne's Hochschild cohomology conjecture. In Recent progress in homotopy theory (Baltimore, MD, 2000), Contemp. Math. 293, Amer. Math. Soc., Providence, RI, 2002, 153-193. Zbl 1009.18009 MR 1890736

[24] A. Nijenhuis, A Lie product for the cohomology of subalgebras with coefficients in the quotient. Bull. Amer. Math. Soc. 73 (1967), 962-967. Zbl 0153.36105 MR 0217152 
[25] S. B. Priddy, Koszul resolutions. Trans. Amer. Math. Soc. 152 (1970), 39-60. Zbl 0261.18016 MR 0265437

[26] D. Tamarkin, Another proof of M. Kontsevich formality theorem. arXiv:math.QA/9803025

[27] D. Tamarkin, Formality of chain operad of little discs. Lett. Math. Phys. 66 (2003), 65-72. Zbl 1048.18007 MR 2064592

[28] M. Van den Bergh, On global deformation quantization in the algebraic case. arXiv:math.AG/0603200

[29] J. Vey, Déformation du crochet de Poisson sur une variété symplectique. Comment. Math. Helv. 50 (1975), 421-454. Zbl 0351.53029 MR 0420753

[30] A. Voronov, Homotopy Gerstenhaber algebras. In Conférence Moshé Flato 1999, Vol. II (Dijon), Math. Phys. Stud. 22, Kluwer Acad. Publ., Dordrecht 2000, 307-331. Zbl 0974.16005 MR 1805923

[31] A. Yekutieli, The continuous Hochschild cochain complex of a scheme. Canad. J. Math. 54 (2002), 1319-1337. Zbl 1047.16004 MR 1940241

[32] A. Yekutieli, Mixed resolutions, simplicial sections and unipotent group actions. arXiv:math.AG/0502206

Received May 19, 2006; revised October 10, 2006

Department of Mathematics, Northwestern University, Evanston, IL 60208, U.S.A.

E-mail: \{vald, tamarkin, tsygan $\} @$ math.northwestern.edu 